ORIGINAL ARTICLE

\title{
Assessment of the Need for Early Initiation of Rehabilitation Treatments in Patients with Coronavirus Disease 2019
}

\author{
Emily Suzuki, MD Tomoko Sakai, MD, PhD Chisato Hoshino, MD, PhD Masanobu Hirao, MD \\ Reiko Yamaguchi, MD, PhD and Rui Nakahara, MD
}

\begin{abstract}
Objective: The aim of this study was to describe the clinical characteristics of coronavirus disease (COVID-19) patients, including risk factors for deep vein thrombosis and pulmonary embolism, and to evaluate the need for rehabilitation to prevent pulmonary embolism. Methods: A retrospective medical record review was conducted of patients admitted to the study hospital with COVID-19 between April 2 and April 23, 2020. The clinical characteristics and blood test results of patients with no history on admission of oral anticoagulant use were evaluated to assess the importance of inflammation and clotting function as risk factors for pulmonary embolism. Results: A total of 51 patients with COVID-19 were admitted during the study period. Their median age was 54.0 years (range: $41-63$ years) and 38 of 51 (74.5\%) were men. The most common comorbidities in men were diabetes $(9 / 38,23.7 \%)$ and hypertension $(13 / 38,34.2 \%)$. On admission, white blood cell counts were normal in both sexes, whereas C-reactive protein and hemostatic marker levels, except for the activated partial thromboplastin time, were significantly higher in men. Moreover, C-reactive protein and hemostatic marker levels were significantly higher in patients that required invasive ventilation. Two patients were diagnosed with acute pulmonary embolism, neither of whom required invasive ventilation. Conclusions: Hypercoagulability and hyperinflammation were observed in COVID-19 patients, especially in men with high oxygen demand. We recommend anticoagulant therapy and early rehabilitation intervention to prevent pulmonary embolism in COVID-19 patients.
\end{abstract}

Key Words: Acute pulmonary embolism; COVID-19; early intervention; hypercoagulability; rehabilitation

\section{INTRODUCTION}

Since the first confirmed patient with coronavirus disease (COVID-19) was identified in Wuhan, China, in December 2019, the number of patients with COVID-19 has rapidly increased worldwide. In Japan, especially in densely populated Tokyo, the number of patients has far exceeded the capacity of hospitals designated for infectious diseases, and, as a result, non-designated hospitals began accepting patients with COVID-19 in early April 2020. Our hospital, Tokyo Medical and Dental University, Medical Hospital, located in the center of Tokyo, started accepting patients with COVID-19 from April 2, which was before the Japanese government de- clared a nationwide state of emergency amid the coronavirus outbreak on April 7. Our hospital provides medical care for patients with COVID-19 who require or are likely to require oxygen supplementation.

Resources for rehabilitation of patients with COVID-19 are limited, and no report has described the rehabilitation of moderate to severe COVID-19 patients in the acute phase. Guidelines on the management of COVID-19 in the acute hospital setting recommend physiotherapy only for patients with significant functional decline and/or at risk of intensive care unit-acquired weakness. ${ }^{1)}$ One report recommended rehabilitation to prevent or to recover from post-intensive care syndrome only for patients who are projected to survive

Received: June 14, 2020, Accepted: July 28, 2020, Published online: August 13, 2020

Department of Rehabilitation Medicine, Tokyo Medical and Dental University, Tokyo, Japan

Correspondence: Tomoko Sakai, MD, PhD, 1-5-45 Yushima, Bunkyo-ku, Tokyo 113 - 8510, Japan, E-mail: t_sakai.orth@tmd.ac.jp

Copyright (C) 2020 The Japanese Association of Rehabilitation Medicine

This is an open-access article distributed under the terms of the Creative Commons Attribution Non-Commercial No

Derivatives (CC BY-NC-ND) 4.0 License. http://creativecommons.org/licenses/by-nc-nd/4.0/ 
Patients with COVID-19 hospitalized at Tokyo Medical and Dental University

from April 2, 2020, to April 23, $2020(n=51)$

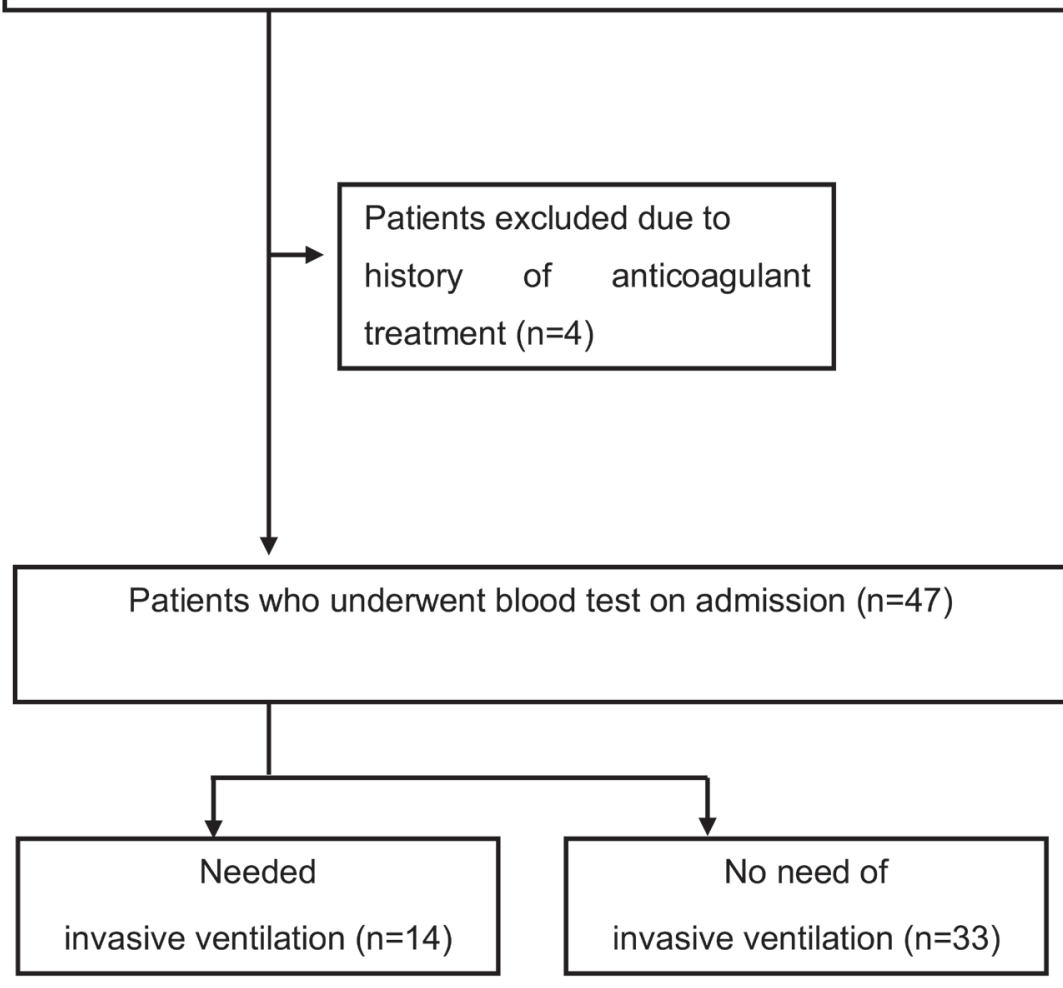

Fig. 1. Patient selection flowchart.

the acute respiratory phase. ${ }^{2)}$ Our hospital did not plan to facilitate rehabilitation during the early phase in moderately or severely ill patients, but, rather, to provide rehabilitation for COVID-19 survivors. The reasons for this approach were to avoid hospital-acquired infections and to reduce the need for personal protective equipment (PPE). However, unexpectedly, we experienced two cases of acute pulmonary embolism (PE) during the early phase of treatment in patients with moderate COVID-19, suggesting the need for early rehabilitation intervention in patients with COVID-19. Because Japan did not experience an explosive increase of COVID-19 hospital admissions or a critical shortage of PPE, the timing of when to start rehabilitation interventions in COVID-19 patients needs to be determined.

However, data on the clinical features of patients with COVID-19 at risk of PE and the corresponding rehabilitation measures are currently limited. Recent studies have reported hypercoagulability and hyperinflammation in COVID-19 patients. ${ }^{3,4)}$ Although some reports have emphasized the importance of anticoagulation therapy in COVID-19 patients, ${ }^{5-7)}$ none have discussed the role of early rehabilitation intervention. This study aimed to investigate the characteristics of patients with COVID-19 in Japan, particularly with respect to the risk factors for PE prior to rehabilitation intervention, and to evaluate the necessity of rehabilitation in the early phase of treatment.

\section{MATERIALS AND METHODS}

\section{Study Design and Subjects}

This retrospective study included 51 consecutive patients with COVID-19 who were admitted to the Tokyo Medical and Dental University, Medical Hospital, Tokyo, Japan, between April 2, 2020, and April 23, 2020. Figure 1 illustrates the patient selection process. The diagnosis of COVID-19 was confirmed using real-time fluorescence reverse transcription polymerase chain reaction (RT-PCR) analysis of nasopharyngeal swabs (Cat No. 204445, QuantiTect Probe RT-PCR Kit, Qiagen, Hilden, Germany). All patients could perform full activities of daily living (ADL) before the infection. Data were collected from medical charts to analyze the patients' characteristics, including their risk of venous 
thromboembolism according to the guidelines of the British Thoracic Society 2003. ${ }^{8}$ ) Because hypercoagulability and hyperinflammation have been reported in patients with COVID-19, we compared coagulability and inflammation by sex and the severity of COVID-19 to identify risk factors for PE that can be used to determine the necessity of early rehabilitation intervention to prevent PE. Four patients who had been taking an anticoagulant were excluded from the evaluation of coagulability. In our hospital, invasive ventilation was performed with a 5-1/min oxygen mask when $\mathrm{SpO}_{2}$ decreased below $90 \%$. However, there were some cases in which invasive ventilation was performed in advance because the possibility of the patient becoming severely ill was considered to be high based on computed tomography (CT) imaging results and the presence of underlying risk factors. Our hospital did not use noninvasive positive pressure ventilation because it can result in air leaks of $30 \mathrm{~L} / \mathrm{min}$, which increase the risk of aerosol transmission.

\section{Measurements}

Blood test parameters, including white blood cell (WBC) counts, prothrombin time (PT), activated partial thromboplastin time (aPTT), fibrinogen (Fbg), and D-dimer levels, were measured using a CS-5100 automated coagulation analyzer (Sysmex Corporation, Kobe, Japan), whereas C-reactive protein (CRP) levels were measured using a LABOSPECT analyzer (Hitachi High-Tech Corporation, Tokyo, Japan).

\section{Statistical Analysis}

The Mann-Whitney U test was used to compare clinical and demographic variables between the sexes, e.g., age, body mass index (BMI), and the time between symptom onset and diagnosis. This test was also used to compare blood test results such as WBC counts, prothrombin time-international normalized ratio (PT-INR), aPTT, Fbg, D-dimer, and CRP levels by sex and by the necessity of invasive ventilation. The chi-squared test was used to compare obesity, smoking history, diabetes, hypertension, and anticoagulant usage between the sexes. All statistical analyses were performed using the software EZR, which is a graphical user interface for $\mathrm{R}$ (The $\mathrm{R}$ foundation for Statistical Computing, Vienna, Austria). ${ }^{9)} \quad \mathrm{P}<0.05$ was considered statistically significant. This study was quickly approved by the Research Ethics Committee of the Tokyo Medical and Dental University using a fast-track process. All procedures performed in this study were in accordance with the Declaration of Helsinki. Oral informed consent was obtained from all patients, and written informed consent was waived from the viewpoint of infection prevention.

\section{RESULTS}

\section{Patient Characteristics}

Of the 51 subjects, 38 were men and 13 were women. The median patient age was 54.0 (range: 41-63) years at the time of RT-PCR for severe acute respiratory syndrome coronavirus 2 (SARS-CoV-2) detection (Table 1). The male patients had a significantly higher BMI than did the female patients. The male patients also tended to be older and to have higher rates of smoking history and diabetes than did female patients, although the differences were not significant between the two groups. The rates of hypertension and ongoing oral anticoagulant therapy were also not significantly different between the male and the female patients. The median time between symptom onset and diagnosis was 7 (range: 5-9) days in the entire group, with no significant difference between the male and female patients.

\section{Comparison of Inflammation and Coagulability According to Sex}

WBC counts were normal in both sexes. PT-INR and CRP, Fbg, and D-dimer levels were significantly higher in men than in women, whereas there was no significant difference in aPTT. Accordingly, hypercoagulability was more likely to be observed in male patients (Table 2).

\section{Comparison of Inflammation and Coagulability According to the Need for Invasive Ventilation}

The WBC count, as an indicator of inflammation, was normal in both groups (i.e., the 33 patients who did not undergo invasive ventilation vs. the 14 patients who did undergo invasive ventilation), but the CRP level was significantly higher in patients who needed invasive ventilation during treatment (Table 3). PT-INR, aPTT, and D-dimer levels were also significantly higher in those who required invasive ventilation than in those who did not.

\section{Two Cases of Acute Pulmonary Embolism}

The clinical course, including the sequential changes in D-dimer levels after admission, of the two patients who developed acute PE are summarized in Fig. 2. The case details are presented below.

\section{Case 1}

A moderately overweight 67-year-old man (BMI $25.1 \mathrm{~kg} /$ 
Table 1. Patient clinical and demographic characteristics according to sex

\begin{tabular}{lcccc}
\hline & $\begin{array}{c}\text { Total } \\
\mathrm{n}=51\end{array}$ & $\begin{array}{c}\text { Male } \\
\mathrm{n}=38\end{array}$ & $\begin{array}{c}\text { Female } \\
\mathrm{n}=13\end{array}$ & P-value $^{\mathrm{d}}$ \\
\hline Age, years & $54.0(41-63)$ & $55.0(47-64)$ & $35.0(30-63)$ & 0.0896 \\
Weight $^{\mathrm{a}}, \mathrm{kg}$ & $72.0(67.0-80.0)$ & $75.0(70.5-82.8)$ & $49.5(44.5-53.8)$ & 0.000246 \\
Height $^{\mathrm{a}}, \mathrm{cm}$ & $172(165-178)$ & $179(173-190)$ & $159(156-159.8)$ & 0.000203 \\
BMI $^{\mathrm{a}, \mathrm{b}}, \mathrm{kg} / \mathrm{m}^{2}$ & $24.7(21.8-26.4)$ & $24.9(23.7-26.5)$ & $19.2(17.6-21.2)$ & 0.00820 \\
Time between symptom onset & $7(5-9)$ & $7(5-8)$ & $8(4-13)$ & 0.479 \\
and diagnosis, days & & & & 0.0504 \\
& & & 36.4 & 0 \\
Smoking historyc, \% & 61.0 & 70.0 & 23.7 & 0.0530 \\
Diabetes, \% & 17.6 & 34.2 & 7.69 & 0.455 \\
Hypertension, \% & 31.4 & 7.89 & & 0.981 \\
History of anticoagulant treat- & 7.84 & & & \\
ment, \% & & & & \\
\hline
\end{tabular}

Data are presented as median (range).

${ }^{a}$ Among the 51 patients, body weight and body height data were available for only 33 patients (27 men and 6 women).

${ }^{b}$ Obesity is defined as BMI $\geq 25 \mathrm{~kg} / \mathrm{m}^{2}$ according to the Japan Society for the Study of Obesity.

${ }^{\mathrm{c}}$ Among the 51 patients, smoking history data were available for only 41 patients (30 men and 11 women).

${ }^{\mathrm{d}}$ The P-values for baseline characteristics were calculated using the Mann-Whitney U test for comparison by sex. A chisquare test was used for obesity, smoking history, diabetes, hypertension, and history of intake treatment.

Table 2. Blood test results on admission according to sex

\begin{tabular}{lcccc}
\hline & $\begin{array}{c}\text { Total } \\
(\mathrm{n}=47)\end{array}$ & $\begin{array}{c}\text { Male } \\
(\mathrm{n}=35)\end{array}$ & $\begin{array}{c}\text { Female } \\
(\mathrm{n}=12)\end{array}$ & P-value $^{\mathrm{b}}$ \\
\hline WBC,$\times 10^{3} / \mu \mathrm{l}$ & $5.70(4.45-7.30)$ & $5.90(4.45-7.35)$ & $5.45(4.85-6.73)$ & 0.502 \\
PT, $\mathrm{s}$ & $11.7(11.0-12.6)$ & $11.9(11.2-12.9)$ & $11.0(10.5-11.6)$ & 0.00967 \\
PT, \% & $82.2(73.0-89.9)$ & $79.1(71.5-86.2)$ & $89.3(84.1-96.4)$ & 0.0142 \\
PT/INR & $1.12(1.06-1.19)$ & $1.13(1.08-1.22)$ & $1.06(1.01-1.11)$ & 0.0104 \\
aPTT, s & $32(29.6-35.5)$ & $32.4(30.8-35.6)$ & $30.8(28.2-32.4)$ & 0.121 \\
Fibrinogen ${ }^{\mathrm{a}}, \mathrm{mg} / \mathrm{dl}$ & $509(407-578)$ & $554(494-609)$ & $342(282-435)$ & 0.0000684 \\
D-dimer, $\mu \mathrm{g} / \mathrm{ml}$ & $0.76(0.36-1.52)$ & $0.92(0.51-2.13)$ & $0.38(0.31-0.53)$ & 0.013 \\
CRP, mg/l & $6.06(1.60-10.5)$ & $7.72(3.94-11.8)$ & $0.38(0.02-2.41)$ & 0.0000992 \\
\hline
\end{tabular}

Data are presented as the median (range).

The median duration between symptom onset and diagnosis was 10 (range, 8-13) days.

${ }^{a}$ Fibrinogen data were obtained for only the 42 patients (30 men and 12 women) who underwent the test.

${ }^{b}$ The P-value for blood test results was calculated using the Mann-Whitney U test for comparison by sex.

WBC, white blood cell; PT, prothrombin time; INR, international normalized ratio; aPTT, activated partial thromboplastin time; CRP, C-reactive protein.

$\mathrm{m}^{2}$ ) who formerly smoked 100 cigarettes per day for 20 years presented with cough and fever that started 13 days prior to admission. He had been bedridden at home for more than 10 days since he had tested positive for SARS-CoV-2 at a nearby clinic. The day before the patient was admitted to our hospital, he sought a consultation at a public health center because of the onset of malaise, dyspnea, and dysgeusia. He was accordingly advised to visit our hospital. At admission, the patient's temperature was $36.6^{\circ} \mathrm{C}$ and the oxygen satura- tion was $84 \%$ on room air. Laboratory test results showed a normal WBC count $\left(5.50 \times 10^{3} / \mu \mathrm{l}\right)$ but elevated CRP $(28.8 \mathrm{mg} /$ dl) and D-dimer $(93 \mu \mathrm{g} / \mathrm{ml})$ levels. Contrast-enhanced CT performed on hospital day 4 for suspicion of PE based on the persistently high serum D-dimer level $(72.2 \mu \mathrm{g} / \mathrm{ml})$ revealed acute bilateral PE. Rivaroxaban $15 \mathrm{mg}$ twice daily and intravenous heparin (5000 units) were administered on hospital day 5 and the D-dimer level gradually decreased (Fig 2A). The patient did not require invasive ventilation during hos- 
Table 3. Blood test results on admission according to the need for invasive ventilation

\begin{tabular}{|c|c|c|c|c|c|c|}
\hline & $\begin{array}{c}\text { Total } \\
(n=47)\end{array}$ & $\begin{array}{l}\text { No need of invasive } \\
\text { ventilation } \\
(n=33)\end{array}$ & $\begin{array}{l}\text { Needed invasive } \\
\text { ventilation } \\
(n=14)\end{array}$ & P-value ${ }^{b}$ & Case 1 & Case 2 \\
\hline $\mathrm{WBC}, \times 10^{3} / \mu \mathrm{l}$ & $5.70(4.45-7.30)$ & $5.60(4.30-7.20)$ & $6.25(4.68-7.38)$ & 0.569 & 5.50 & 5.9 \\
\hline $\mathrm{PT}, \mathrm{s}$ & $11.7(11.0-12.6)$ & $11.5(10.6-12.1)$ & $12.7(11.6-13.2)$ & 0.00486 & 16.7 & 11.2 \\
\hline PT, $\%$ & $82.2(73.0-89.9)$ & $72.9(67.5-83.4)$ & 85.5 (79.0-95.7) & 0.00453 & 47.8 & 85.6 \\
\hline PT/INR & $1.12(1.06-1.19)$ & $1.10(1.02-1.15)$ & $1.20(1.11-1.24)$ & 0.00468 & 1.53 & 1.08 \\
\hline aPTT, s & $32(29.6-35.5)$ & $31.3(29.1-33.2)$ & $35.4(33.6-37.7)$ & 0.00627 & 32 & 33.9 \\
\hline Fibrinogen $^{\mathrm{a}}, \mathrm{mg} / \mathrm{dl}$ & $509(407-578)$ & $463(359-575)$ & $555(535-573)$ & 0.0641 & 465 & NA \\
\hline D-dimer, $\mu \mathrm{g} / \mathrm{ml}$ & $0.76(0.36-1.52)$ & $0.60(0.34-1.34)$ & $1.21(0.75-4.25)$ & 0.0253 & 93 & 7.72 \\
\hline $\mathrm{CRP}, \mathrm{mg} / \mathrm{l}$ & $6.06(1.60-10.5)$ & $2.86(0.39-9.30)$ & $9.28(6.25-17.7)$ & 0.00406 & 28.77 & 10.49 \\
\hline
\end{tabular}

Data are presented as median (range).

The period from onset to blood test on admission was 10 (8-13) days.

The median time between symptom onset and diagnosis was 5 (3-11) days.

${ }^{\text {a}}$ Fibrinogen data were obtained for only the 42 patients ( 31 among patients who did not need invasive ventilation and 11 among patients who required invasive ventilation) who underwent the test.

${ }^{b}$ The Mann-Whitney U test was performed between those needing and not needing invasive ventilation.

All 14 patients who required invasive ventilation were male.

pitalization.

\section{Case 2}

An overweight 56 -year-old man (BMI: $32.7 \mathrm{~kg} / \mathrm{m}^{2}$ ) who had never smoked presented with malaise that had started 6 days prior to his visit to our hospital. The medical history included type 2 diabetes, hyperuricemia, dyslipidemia, and colostomy for rectal cancer. The patient tested positive for SARS-CoV-2 in an RT-PCR test at a local clinic 4 days prior to admission and he was referred to our hospital by the public health center. At admission, the patient's temperature was $37.5^{\circ} \mathrm{C}$ and the oxygen saturation was $93 \%$ on room air. On day 3 , his oxygen level decreased to $90 \%$ with $21 /$ min oxygen by nasal canulae with complaint of breathlessness on sitting. Contrast-enhanced CT on hospital day 7 because of the continuous high oxygen requirement revealed acute bilateral PE. The D-dimer level decreased after apixaban $20 \mathrm{mg}$ daily was started on day 10. The patient did not require invasive ventilation during hospitalization.

\section{DISCUSSION}

In the current study, male patients tended to be older, have a higher BMI, and have higher rates of smoking history and diabetes than female patients. Previous reports have indicated that obesity, older age, smoking history, and diabetes are risk factors for severe COVID-19.10,11) This implies that our male patients tended to have more risk factors for severe COVID-19 at baseline. Our findings showed that hypercoagulability and hyperinflammation were observed in patients with COVID-19, especially in men with high oxygen demand. Considering that inactivity is a risk for blood clots and PE regardless of the underlying disease, ${ }^{12,13)}$ early rehabilitation intervention in addition to anticoagulant therapy is recommended to prevent PE in patients with COVID-19. In this study, most of the infected patients were men (74.5\%), less than half of whom had diabetes (23.7\%) and hypertension $(34.2 \%)$, which is consistent with the results of other recent reports. ${ }^{14,15)}$ The median age of the overall population was slightly higher (54.0 [41-63] years) than that in these previous reports (49 [41-58] and 47 [35-58] years, respectively). Considering that the aging population in Japan (i.e., citizens aged more than 65 years) is the largest worldwide, accounting for $28.1 \%$ of the Japanese population on October $1,2018,{ }^{16)}$ in contrast with $10.9 \%$ in China and $16 \%$ in the United States, ${ }^{17)}$ physical and pulmonary rehabilitation for elderly COVID-19 patients will be crucial to help maintain pre-hospitalization ADL performance levels.

When assessing clotting function and inflammatory markers, PT-INR, Fbg, and D-dimer levels increased in parallel with the CRP level, all of which were significantly higher in male patients than in female patients. Furthermore, hypercoagulability and hyperinflammation were observed in those who subsequently required invasive ventilation. These findings indicate that hyperinflammation and hypercoagulability are more likely to be observed in male patients who require more than $5 \mathrm{l} / \mathrm{min}$ oxygen during treatment. Because hypercoagulability is a cause of $\mathrm{PE}{ }^{18)}$ the results of this study may 

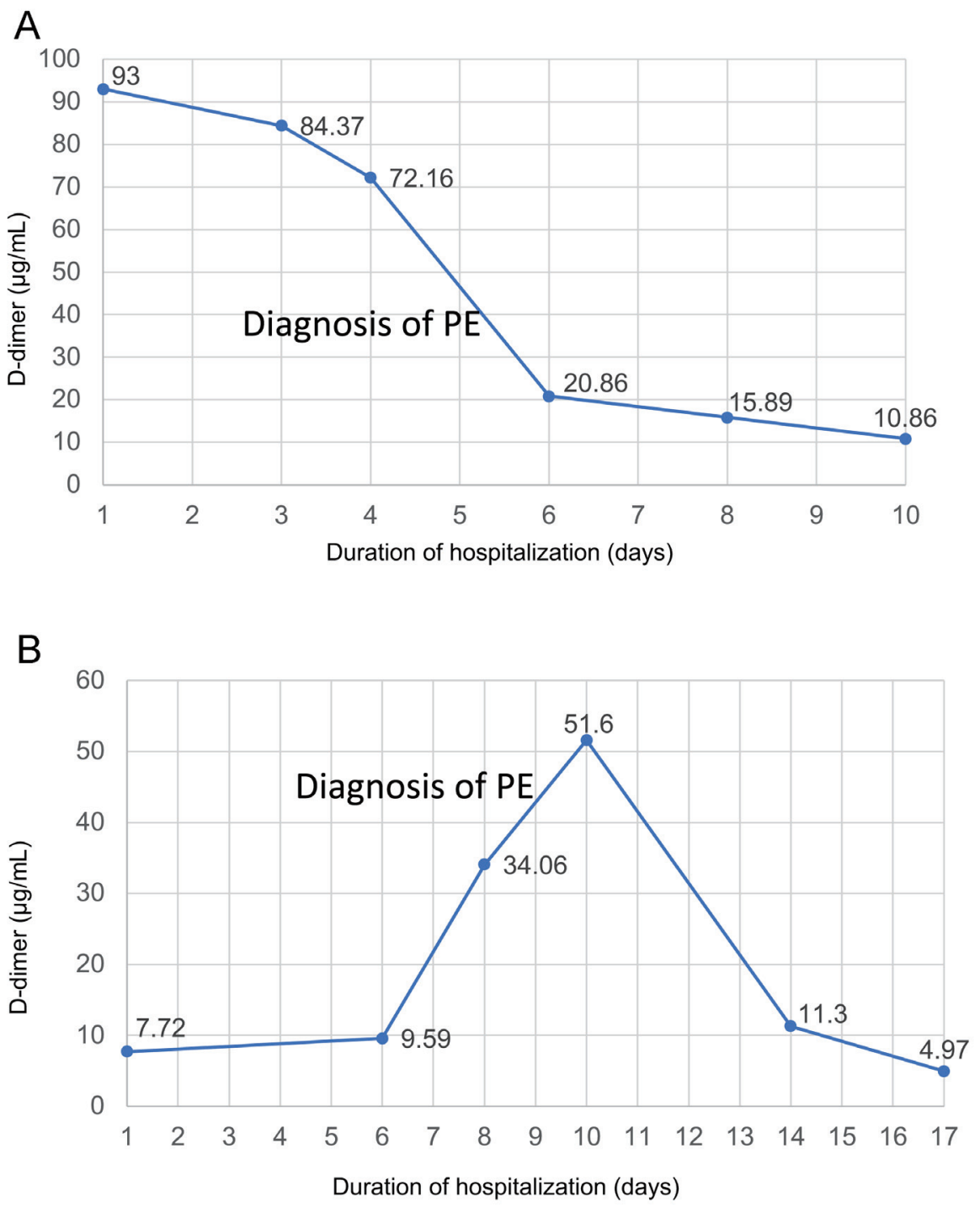

Fig. 2. Changes in D-dimer levels after admission. (A) Case 1: Pulmonary embolism (PE) was found on contrast-enhanced $\mathrm{CT}$ on day 4, and the D-dimer level decreased after rivaroxaban $15 \mathrm{mg}$ twice daily was started and intravenous administration of heparin (5000 units) on day 5. (B) Case 2: PE was diagnosed via contrast-enhanced CT on day 7, and the D-dimer level decreased after starting apixaban $20 \mathrm{mg}$ daily on day 10.

imply that male patients who require oxygen supplementation are at a high risk of PE and should be prioritized for early mobilization. Nonetheless, some patients who did not require invasive ventilation also displayed hypercoagulability. Furthermore, the two patients who developed PE did not require invasive ventilation during hospitalization. Consequently, the promotion of early mobilization of patients with COVID-19 should not be limited to those with severe disease.

In this study, symptomatic PE occurred in 2 of 51 patients with COVID-19, yielding a prevalence rate of $3.9 \%$. This is markedly higher than the prevalence of PE after total knee arthroplasty without chemoprophylaxis in the Asian popula- tion $(0.01 \%){ }^{19)}$ One of the reasons for this high prevalence of $\mathrm{PE}$ is unique to COVID-19 in that it causes hypercoagulability and hyperinflammation. ${ }^{3)}$ Another possible reason is that patients with COVID-19 tend to move less because of dyspnea and the limited room space during self-isolation. The incidence of symptomatic PE, let alone asymptomatic PE, is likely to be higher than reported because the symptoms and signs of pulmonary embolism (dyspnea and coagulopathy) are difficult to distinguish from the signs and symptoms of COVID-19 itself. ${ }^{14,20)}$ Moreover, both contrast-enhanced CT for suspected PE and ultrasonography for venous thrombosis are likely to be avoided in COVID-19 patients to minimize 
infection transmission.

Evidently, the promotion of mobilization as well as anticoagulant therapy to prevent deep vein thrombosis (DVT) and PE are important. Currently, in our hospital, either apixaban or rivaroxaban is selected as an anticoagulant depending on each doctor's preference. Considering the high risk of hospital-acquired infections and the worldwide shortage of medical resources, including not only PPE but also physicians, nurses, and rehabilitation therapists, it is understandable that the guidelines for physiotherapy do not recommend affirmative intervention for patients with moderate to severe COVID-19. However, if medical resources are available, physiotherapy should be considered in all patients with $\mathrm{CO}$ VID-19, regardless of the severity of the disease. Remote rehabilitation could be an option to prevent $\mathrm{PE}$ in patients with mild disease who do not require oxygen therapy or those who can fully perform ADL. Remote rehabilitation reduces both close contact with COVID-19 patients and the need for PPE. Another possible reason for not promoting rehabilitation in severe COVID-19 patients is that rehabilitation treatment options are quite limited for patients under invasive ventilation. Nevertheless, we believe that rehabilitation interventions should be promoted because physical rehabilitation from the onset of mechanical ventilation has been reported to be beneficial to patients' clinical outcomes. ${ }^{21)}$

The risk management of PE in COVID-19 patients is especially important. The presence of DVT should be suspected in patients whose D-dimer and CRP levels are high. To minimize the risk of PE occurring as a result of DVT, the use of anticoagulant therapy and imaging examinations are crucial. Under normal circumstances, enhanced CT and ultrasound by vascular specialists are the first choices to rule out proximal DVT. However, the transfer of COVID-19 patients to the examination room carries a risk of transmitting infection. Therefore, we recommend the use of three-point compression ultrasonography by an attending physician in an isolation ward for COVID-19 patients with high D-dimer levels. Mousavi-Roknabadi et al. ${ }^{22)}$ reported that three-point compression ultrasonography identified the presence of lower-extremity DVT with an accuracy of $96.4 \%$ in comparison with whole-leg compression ultrasound. Moreover, three-point compression ultrasonography showed high specificity regardless of the skill of the clinician performing the investigation. ${ }^{23)}$ Furthermore, the short amount of time that is required to perform three-point compression ultrasound reduces the risk of transmission of infection. ${ }^{24)}$

Our study has several limitations. First, this was a singlecenter, short-duration, retrospective, observational study. A future study covering a longer period is necessary to validate the risk and effect of mobilization for DVT and PE in COVID-19. However, although only a small number of patients were included in the analysis, we still believe that it is meaningful to reveal the clinical characteristics of patients with COVID-19 in Japan and share our lessons on the urgent need of rehabilitation. We are planning in the near future to report the outcomes of PE prevalence after we began rehabilitation in our hospital from April 24. Second, not all patients underwent contrast-enhanced $\mathrm{CT}$ to rule out $\mathrm{PE}$, and therefore the true prevalence of $\mathrm{PE}$ in our hospital could have been higher. Third, although rehabilitation is recommended, it is unclear whether early rehabilitation is effective for preventing PE. Further study is necessary to determine whether early rehabilitation can prevent PE in COVID-19 patients.

In conclusion, the hypercoagulability and hyperinflammation observed in patients with COVID-19 predisposes them to PE. Early rehabilitation intervention is recommended not only to facilitate respiratory function and recovery from muscle weakness but also to prevent PE.

\section{ACKNOWLEDGMENTS}

We thank all members of the Department of Integrated Pulmonology and Trauma and Acute Critical Care Center, Tokyo Medical and Dental University, for their dedicated treatment of patients with COVID-19.

\section{CONFLICTS OF INTEREST}

The authors declare that they have no conflict of interests.

\section{REFERENCES}

1. Thomas P, Baldwin C, Bissett B, Boden I, Gosselink R, Granger CL, Hodgson C, Jones AY, Kho ME, Moses R, Ntoumenopoulos G, Parry SM, Patman S, van der Lee L: Physiotherapy management for COVID-19 in the acute hospital setting: clinical practice recommendations. J Physiother 2020;66:73-82. DOI:10.1016/j. jphys.2020.03.011, PMID:32312646

2. Stam H, Stucki G, Bickenbach J: Covid-19 and post intensive care syndrome: a call for action. J Rehabil Med 2020;52:jrm00044. DOI:10.2340/16501977-2677, PMID:32286675 
3. Spiezia L, Boscolo A, Poletto F, Cerruti L, Tiberio I, Campello E, Navalesi P, Simioni P: COVID19-related severe hypercoagulability in patients admitted to intensive care unit for acute respiratory failure. Thromb Haemost 2020;120:998-1000. DOI:10.1055/s-0040-1710018, PMID:32316063

4. Panigada M, Bottino N, Tagliabue P, Grasselli G, Novembrino C, Chantarangkul V, Pesenti A, Peyvandi F, Tripodi A: Hypercoagulability of COVID-19 patients in intensive care unit: a report of thromboelastography findings and other parameters of hemostasis. J Thromb Haemost 2020;18:1738-1742. DOI:10.1111/jth.14850, PMID:32302438

5. Tang N, Bai H, Chen X, Gong J, Li D, Sun Z: Anticoagulant treatment is associated with decreased mortality in severe coronavirus disease 2019 patients with coagulopathy. J Thromb Haemost 2020;18:1094-1099. DOI:10.1111/jth.14817, PMID:32220112

6. Ranucci M, Ballotta A, Di Dedda U, Bayshnikova E, Dei Poli M, Resta M, Falco M, Albano G, Menicanti L: The procoagulant pattern of patients with COVID-19 acute respiratory distress syndrome. J Thromb Haemost 2020;18:1747-1751. DOI:10.1111/jth.14854, PMID:32302448

7. Li T, Lu H, Zhang W: Clinical observation and management of COVID-19 patients. Emerg Microbes Infect 2020;9:687-690. DOI:10.1080/22221751.2020.17 41327, PMID:32208840

8. British Thoracic Society Standards of Care Committee Pulmonary Embolism Guideline Development Group: British Thoracic Society guidelines for the management of suspected acute pulmonary embolism. Tho$\operatorname{rax}$ 2003;58:470-483. DOI:10.1136/thorax.58.6.470, PMID:12775856

9. Kanda Y: Investigation of the freely available easyto-use software 'EZR' for medical statistics. Bone Marrow Transplant 2013;48:452-458. DOI:10.1038/ bmt.2012.244, PMID:23208313

10. Zheng Z, Peng F, Xu B, Zhao J, Liu H, Peng J, Li Q, Jiang C, Zhou Y, Liu S, Ye C, Zhang P, Xing Y, Guo H, Tang W: Risk factors of critical \& mortal COVID-19 cases: a systematic literature review and meta-analysis. J Infect 2020;81:e16-e25. DOI:10.1016/j. jinf.2020.04.021, PMID:32335169
11. Zheng KI, Gao F, Wang XB, Sun QF, Pan KH, Wang TY, Ma HL, Chen YP, Liu WY, George J, Zheng MH: Letter to the Editor: Obesity as a risk factor for greater severity of COVID-19 in patients with metabolic associated fatty liver disease. Metabolism 2020;108:154244. doi: DOI:10.1016/j.metabol.2020.154244.

12. Shirakawa T, Iso H, Yamagishi K, Yatsuya H, Tanabe N, Ikehara S, Ukawa S, Tamakoshi A: Watching television and risk of mortality from pulmonary embolism among Japanese men and women. Circulation 2016;134:355357. DOI:10.1161/CIRCULATIONAHA.116.023671, PMID:27462056

13. Kabrhel C, Varraso R, Goldhaber SZ, Rimm E, Camargo CA Jr: Physical inactivity and idiopathic pulmonary embolism in women: prospective study. BMJ 2011;343(jul04 1):d3867. DOI:10.1136/bmj.d3867, PMID:21727169

14. Huang C, Wang Y, Li X, Ren L, Zhao J, Hu Y, Zhang L, Fan G, Xu J, Gu X, Cheng Z, Yu T, Xia J, Wei Y, Wu W, Xie X, Yin W, Li H, Liu M, Xiao Y, Gao H, Guo L, Xie J, Wang G, Jiang R, Gao Z, Jin Q, Wang J, Cao B: Clinical features of patients infected with 2019 novel coronavirus in Wuhan, China. Lancet 2020;395:497-506. DOI:10.1016/S0140-6736(20)301835, PMID:31986264

15. Guan W, Ni Z, Hu Y, Liang W, Ou C, He J, Liu L, Shan H, Lei C, Hui DS, Du B, Li L, Zeng G, Yuen KY, Chen R, Tang C, Wang T, Chen P, Xiang J, Li S, Wang J, Liang Z, Peng Y, Wei L, Liu Y, Hu Y, Peng P, Wang J, Liu J, Chen Z, Li G, Zheng Z, Qiu S, Luo J, Ye C, Zhu S, Zhong N, China Medical Treatment Expert Group for Covid-19: Clinical characteristics of coronavirus disease 2019 in China. N Engl J Med 2020;382:1708-1720. DOI:10.1056/NEJMoa2002032, PMID:32109013

16. Japan Cabinet Office: Annual Report on the Ageing Society, June 2019. Cabinet Office, Japan, 2019.

17. United Nations Department of Economic and Social Affairs: World Bank staff estimates based on age/sex distributions of United Nations Population Division's World Population Prospects: 2019 Revision. United Nations, New York, 2019.

18. Chow V, Reddel C, Pennings G, Chung T, Ng AC, Curnow J, Kritharides L: Persistent global hypercoagulability in long-term survivors of acute pulmonary embolism. Blood Coagul Fibrinolysis 2015;26:537544. DOI:10.1097/MBC.0000000000000285, PMID:25811450 
19. Lee WS, Kim KI, Lee HJ, Kyung HS, Seo SS: The incidence of pulmonary embolism and deep vein thrombosis after knee arthroplasty in Asians remains low: a meta-analysis. Clin Orthop Relat Res 2013;471:1523-1532. DOI:10.1007/s11999-012-2758-9, PMID:23264001

20. Rotzinger DC, Beigelman-Aubry C, von Garnier C, Qanadli SD: Pulmonary embolism in patients with COVID-19: time to change the paradigm of computed tomography. Thromb Res 2020;190:58-59. DOI:10.1016/j. thromres.2020.04.011, PMID:32302782

21. Schweickert WD, Pohlman MC, Pohlman AS, Nigos C, Pawlik AJ, Esbrook CL, Spears L, Miller M, Franczyk M, Deprizio D, Schmidt GA, Bowman A, Barr R, McCallister KE, Hall JB, Kress JP: Early physical and occupational therapy in mechanically ventilated, critically ill patients: a randomised controlled trial. Lancet 2009;373:1874-1882. DOI:10.1016/S01406736(09)60658-9, PMID:19446324
22. Mousavi-Roknabadi RS, Dehbozorgi A, Damghani F, Sharifi M, Sajjadi S, Hosseini-Marvast S: Accuracy of three-point compression ultrasound for the diagnosis of proximal deep-vein thrombosis in emergency department. J Res Med Sci 2019;24:80. DOI:10.4103/jrms. JRMS_1057_18, PMID:31620179

23. Jahanian F, Khatir I, Mostafavi E, Moradi S, Aghamalaki F: Diagnostic accuracy of a three-point compression ultrasonography performed by emergency medicine resident for the diagnosis of deep vein thrombosis: a prospective diagnostic study. Acta Inform Med 2019;27:119-122. DOI:10.5455/aim.2019.27.119-122, PMID:31452570

24. Pedraza García J, Valle Alonso J, Ceballos García P, Rico Rodríguez F, Aguayo López MÁ, MuñozVillanueva MC: Comparison of the accuracy of emergency department-performed point-of-care-ultrasound (POCUS) in the diagnosis of lower-extremity deep vein thrombosis. J Emerg Med 2018;54:656-664. DOI:10.1016/j.jemermed.2017.12.020, PMID:29306580 